TI 2013-057/III

Tinbergen Institute Discussion Paper

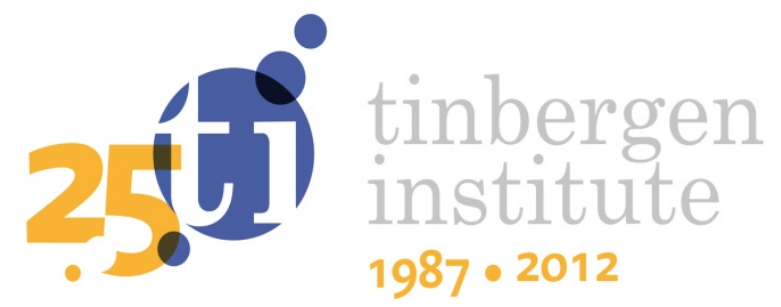

\title{
Analyzing Fixed-Event Forecast Revisions
}

Chia-Lin Changl

Bert de Bruijn²

Philip Hans Franses2

Michael McAleer ${ }^{2}$

\footnotetext{
I National Chung Hsing University Taichung, Taiwan;

2 Econometric Institute, Erasmus School of Economics, Erasmus University Rotterdam, and Tinbergen Institute.
} 
Tinbergen Institute is the graduate school and research institute in economics of Erasmus University Rotterdam, the University of Amsterdam and VU University Amsterdam.

More TI discussion papers can be downloaded at http://www.tinbergen.nl

Tinbergen Institute has two locations:

Tinbergen Institute Amsterdam

Gustav Mahlerplein 117

1082 MS Amsterdam

The Netherlands

Tel.: +31(0)205251600

Tinbergen Institute Rotterdam

Burg. Oudlaan 50

3062 PA Rotterdam

The Netherlands

Tel.: +31(0)10 4088900

Fax: $+31(0) 104089031$

Duisenberg school of finance is a collaboration of the Dutch financial sector and universities, with the ambition to support innovative research and offer top quality academic education in core areas of finance.

DSF research papers can be downloaded at: http://www.dsf.nl/

Duisenberg school of finance

Gustav Mahlerplein 117

1082 MS Amsterdam

The Netherlands

Tel.: +31(0)20 5258579 


\title{
Analyzing Fixed-event Forecast Revisions*
}

\author{
Chia-Lin Chang \\ Department of Applied Economics \\ Department of Finance \\ National Chung Hsing University \\ Taichung, Taiwan \\ Bert de Bruijn \\ Econometric Institute \\ Erasmus School of Economics \\ Erasmus University Rotterdam \\ and \\ Tinbergen Institute \\ The Netherlands \\ Philip Hans Franses \\ Econometric Institute \\ Erasmus School of Economics \\ Erasmus University Rotterdam \\ and \\ Tinbergen Institute \\ The Netherlands \\ Michael McAleer \\ Econometric Institute \\ Erasmus School of Economics \\ Erasmus University Rotterdam \\ and \\ Tinbergen Institute \\ The Netherlands
}

Second revision: March 2013

* The authors are most grateful for the helpful comments and suggestions of the Editor, an Associate Editor and two referees. For financial support, the first author acknowledges the National Science Council, Taiwan, and the fourth author wishes to thank the Australian Research Council, National Science Council, Taiwan, and the Japan Society for the Promotion of Science. 


\begin{abstract}
It is common practice to evaluate fixed-event forecast revisions in macroeconomics by regressing current forecast revisions on one-period lagged forecast revisions. Under weak-form (forecast) efficiency, the correlation between the current and one-period lagged revisions should be zero. The empirical findings in the literature suggest that this null hypothesis of zero correlation is rejected frequently, where the correlation can be either positive (which is widely interpreted in the literature as "smoothing") or negative (which is widely interpreted as "over-reacting"). We propose a methodology to interpret such non-zero correlations in a straightforward and clear manner. Our approach is based on the assumption that numerical forecasts can be decomposed into both an econometric model and random expert intuition. We show that the interpretation of the sign of the correlation between the current and one-period lagged revisions depends on the process governing intuition, and the current and lagged correlations between intuition and news (or shocks to the numerical forecasts). It follows that the estimated non-zero correlation cannot be given a direct interpretation in terms of smoothing or over-reaction.
\end{abstract}

Keywords: Evaluating forecasts, Macroeconomic forecasting, Rationality, Intuition, Weak-form efficiency, Fixed-event forecasts.

JEL Classifications: C22, C53, E27, E37. 


\section{Introduction}

There is a substantial recent literature on the evaluation of macroeconomic forecasts and, in particular, on forecast revisions. Such revisions involve potential changes in the forecasts for the same fixed event. For example, Consensus Forecasters quote forecasts for the value of an economic variable (such as the inflation rate, unemployment rate, real GDP growth rate) in year T, where the forecast origin starts in January of year T-1. When these forecasts continue through to December in year $\mathrm{T}$, there are 24 forecasts for the same fixed event, and hence there are 23 forecast revisions (or updates).

The literature on forecast revisions deals with the empirical merits of these revisions (see, for example, Lawrence and O'Connor (2000) and Cho (2002)) but, for a larger part, it seems to deal with the properties of the updates themselves (see, for example, the recent study of Dovern and Weisser (2011)). The latter seems to be inspired by the recent availability of databases with detailed information of forecasts quoted by a range of professional forecasters.

In this paper, we contribute to this second stream of literature, that is, an evaluation of the properties of the forecast revisions themselves where, in particular, we show how to interpret a key parameter in an auxiliary testing regression.

In the fixed-event forecast revision literature, numerical forecasts are taken as data. It is not necessarily known how the numerical forecasts were obtained. We denote a forecast given at origin, $t-h$, for an event at the fixed target data, $t$, as

$$
F_{t \mid t-h}
$$

where $h=1, \ldots, H$. Therefore, for each event $t$, we have $H$ forecasts, ranging from a onestep-ahead forecast to an $H$-step-ahead forecast. A (first-order) forecast revision is defined by 


$$
F_{t \mid t-h}-F_{t \mid t-(h+1)}
$$

and it is this type of forecast revision that is the focus of this paper.

A commonly-used method to examine the potential properties of forecast revisions is to use auxiliary testing regressions of the form:

$$
F_{t \mid t-h}-F_{t \mid t-(h+1)}=\alpha+\beta\left(F_{t \mid t-(h+1)}-F_{t \mid t-(h+2)}\right)+\xi_{t, h}
$$

where the value of $\beta$ is of key interest, $h$ runs from 1 to $H$, and the sample size is $H$.

Nordhaus (1987) introduced the concept of weak-form efficiency, which entails that, under such efficiency the correlation between subsequent forecast revisions is zero. In other words, under weak-form efficiency, it should be the case that $\beta=0$ in equation (1). As Nordhaus (1987) was concerned with forecasts from econometric models, it is appropriate to refer to this concept as "weak-form model forecast efficiency", whereby fixed-event forecasts taken one period apart differ only randomly. Thus, there is no discernible improvement in forecasts as the fixed event becomes less distant.

It should be emphasized that equation (1) is solely a testing equation, and is not a model. The sole purpose of equation (1) is to test the null hypothesis of weak-form efficiency, that is $\beta=0$. It must be emphasized that rejection of $\beta=0$ is not synonymous with interpreting equation (1) as an appropriate specification for modelling forecast revisions. If this were the case, then equation (1) would be used to estimate forecast revisions rather than for testing the weak-form efficiency of forecast revisions.

A further point to emphasize is that, as an $\mathrm{AR}(1)$ process for testing purposes, equation (1) exhibits geometric decay, regardless of the sign or magnitude of $\beta$. Therefore, the widely-used interpretations of smoothing and over-reaction based on whether $\beta$ is 
estimated to be positive or negative, respectively, in equation (1), must be taken as inherently flawed.

Interestingly, in various recent studies that have analyzed a range of forecast revisions, it has frequently been found that the null hypothesis $\beta=0$ is rejected (see Table 1). Clements (1997) analyzes the forecasts for GDP and CPI made by the National Institute of Economics and Social Research in the UK. Using 5 different versions of equation (1), Clements (1997) documents an average value of $\beta$ of -0.414 for GDP forecast revisions and of -0.232 for inflation forecast revisions (see Table 1 in Clements (1997)). Isengildina et al. (2006) examine forecasts for crop production concerning corn and soybeans, where the forecasts are provided by the U.S. Department of Agriculture. The authors show that 8 of the 10 estimates of $\beta$ are significantly positive.

Using data on the Consensus Economics Forecasts, Dovern and Weisser (2011) conclude that in only a few cases are the estimated values of $\beta$ significantly different from 0 but, when they are significant, they are predominantly negative. These authors interpret their finding as an indication that forecasters overreact to incoming news. Ager et al. (2009) report for GDP that the null hypothesis $\beta=0$ is rejected, with a mean estimate of 0.309 across 24 cases (namely, 12 countries and 2 methods - see their Table 5). In their Table 6, they report a mean estimate of 0.163 across 24 cases for inflation. Isiklar et al. (2006) examine 18 industrialized countries, reporting pooled estimates of $\beta$ equal to 0.330 . Finally, Ashiya (2006), Loungani (2001), and an early study in Berger and Krane (1985), all report small but positive estimates of $\beta$, and interpret these as indications of forecast smoothing.

In summary, we observe from the literature that the estimates of $\beta$ in equation (1) tend to range from -0.5 to 0.5 and, in a significant number of cases, the null hypothesis that $\beta$ $=0$ is rejected. Therefore, it is important to interpret correctly the meaning of a rejection of $\beta=0$. 
In this paper, we propose a methodology to provide an interpretation of the alternative sign outcomes of $\beta$ arising from equation (1). The new approach is based on our conjecture that available forecasts are typically the concerted outcome of an econometric model-based forecast, $M_{t t-h}$, and of the intuition of an expert (such as a professional forecaster), $v_{t t-h}$ (see, for example, Franses et al. (2011) for substantial empirical evidence regarding this conjecture)

There are various reasons why forecasters may deviate from a pure econometric modelbased forecast. Examples are that forecasters aim to attract attention (see Laster, Bennett and Geoum (1999)), or may have alternative loss functions (see, for example, Capistran and Timmermann (2009)).

In what follows, we use the decomposition of an available numerical forecast, which is taken to be the underlying variable of interest, as

$$
F_{t \mid t-h}=M_{t \mid t-h}+v_{t \mid t-h}
$$

It will become apparent that changing $M_{t \mid t-h}$ into $\pi M_{t \mid t-h}$, with $0<\pi<1$, whereby the model forecast may be down-weighted by the expert, does not change the discussion appreciably. Our next step is to propose a model for the intuition $v_{t \mid t-h}$, and to allow for correlation between intuition and the error term $\varepsilon_{t, h}$, in the model. Note that intuition does not need to have mean zero. The interpretation of the sign of the correlation between the current and one-period lagged revisions depends on the process governing intuition, and the correlations between current and one-period lagged intuition and news to the numerical forecast variable. In Section 2 we discuss the methodological approach, and in Section 3 we relate it to the empirical findings in the literature. Section 4 concludes with several further research issues. 


\section{Interpreting the Empirical Findings}

Despite a wealth of empirical evidence on patterns in forecast revisions, to date there would seem to be no studies that have formally analyzed the meaning of positive or negative estimates of $\beta$ in equation (1). If $\beta>0$, there could be some kind of smoothing process that exists, but what type of process might this be? Moreover, what does this smoothing process look like? It is the purpose of this section to propose a formal methodology to derive how specific estimates could arise, where we explicitly take into account that a numerical forecast is a concerted effort of an econometric model and an expert individual's intuition.

We first introduce some notation, and then derive an expression for $\beta$ in equation (1). Finally, we consider several special cases that can be used to explain the observed estimates given in Table 1.

\subsection{Preliminaries}

As stated above, the basic assumption for our methodology is given in (2). We use the familiar Wold decomposition of a stationary time series of interest (namely, the numerical forecasts of key economic fundamentals such as real GDP growth, inflation rate, and unemployment rate), $y_{t}$, that is:

$$
y_{t}=\varepsilon_{t}+\theta_{1} \varepsilon_{t-1}+\theta_{2} \varepsilon_{t-2}+\theta_{3} \varepsilon_{t-3}+\ldots
$$

where $\varepsilon_{t} \sim\left(0, \sigma^{2}\right)$ is an uncorrelated error process. This error process can be interpreted as a news process (as will be seen below). The parameters, $\theta_{k}, k=1,2,3, \ldots$, are such that the time series is stationary and invertible. 
Given (3), the econometric time series model forecasts can be written as $M_{t \mid t-1}=\theta_{1} \varepsilon_{t-1}+\theta_{2} \varepsilon_{t-2}+\theta_{3} \varepsilon_{t-3}+.$. , and so on. Two subsequent forecast updates are given as follows:

$$
\begin{aligned}
F_{t \mid t-1}-F_{t \mid t-2} & =M_{t \mid t-1}-M_{t \mid t-2}+v_{t \mid t-1}-v_{t \mid t-2} \\
& =\theta_{1} \varepsilon_{t-1}+v_{t \mid t-1}-v_{t \mid t-2}
\end{aligned}
$$

and

$$
\begin{aligned}
F_{t \mid t-2}-F_{t \mid t-3} & =M_{t \mid t-2}-M_{t \mid t-3}+v_{t \mid t-2}-v_{t \mid t-3} \\
& =\theta_{2} \varepsilon_{t-2}+v_{t \mid t-2}-v_{t \mid t-3}
\end{aligned}
$$

Note that when $F_{t \mid t-h}=\pi M_{t \mid t-h}+v_{t \mid t-h}$ with $0<\pi<1$, which is the case where the model outcome is only partially taken into account, then similar results will appear as above, as the $\theta$ parameters will then be scaled by $\pi$.

\subsection{Regression parameters}

In order to derive an expression for the regression coefficient in (1) we define the following variances and covariances, which are constant for $i=1,2$, and 3 (and, in general, for $h, h+1$ and $h+2$ ) for expository purposes:

$$
\begin{array}{ll}
\gamma_{0} & \text { variance of } v_{t \mid t-i} \\
\gamma_{1} & \text { covariance between } v_{t \mid t-i} \text { and } v_{t t-(i+1)} \\
\gamma_{2} & \text { covariance between } v_{t t-i} \text { and } v_{t t-(i+2)} \\
\omega_{0} & \text { covariance between } \varepsilon_{t-i} \text { and } v_{t t-i} \\
\omega_{1} & \text { covariance between } \varepsilon_{t-(i+1)} \text { and } v_{t t-i}
\end{array}
$$

The first three terms deal with the time series properties of random expert intuition. The last two terms deal with the potential non-zero correlations between current news and current intuition (namely, how intuition might react contemporaneously to news in the 
numerical forecast), and between one-period lagged news and current intuition (namely, how intuition might react with a one-period lag to news in the numerical forecast). Note that the premise behind forecast smoothing, as it is presented in the literature, is that current news is discarded to some extent, which means that $\omega_{0}<0$.

More precisely, the following definitions will be used to interpret smoothing and overreaction in a clear and meaningful manner:

Definition 1a: Contemporaneous Smoothing of intuition to news occurs when $\omega_{0}<0$

Definition 1b: Dynamic Smoothing of intuition to news occurs when $\omega_{1}<0$

Definition 2a: Contemporaneous Over-reaction of intuition to news occurs when $\omega_{0}>0$

Definition 2b: Dynamic Over-reaction of intuition to news occurs when $\omega_{1}>0$

In light of these definitions, it is possible that $\omega_{0}<0$ and $\omega_{1}>0$, or $\omega_{0}>0$ and $\omega_{1}<0$, so that there can be a switch from smoothing to over-reaction, and vice-versa, over time.

Given the above terms and definitions, we can proceed to show that the variance of $F_{t \mid t-2}-F_{t \mid t-3}$ is equal to

$$
\begin{aligned}
& E\left[\left(\theta_{2} \varepsilon_{t-2}+v_{t \mid t-2}-v_{t t-3}\right)\left(\theta_{2} \varepsilon_{t-2}+v_{t \mid t-2}-v_{t \mid t-3}\right)\right] \\
& \quad=\theta_{2}^{2} \sigma^{2}+2 \theta_{2} \omega_{0}+2 \gamma_{0}-2 \gamma_{1}
\end{aligned}
$$

The covariance between $F_{t t-1}-F_{t \mid t-2}$ and $F_{t t-2}-F_{t t-3}$ is equal to

$$
\begin{gathered}
E\left[\left(\theta_{1} \varepsilon_{t-1}+v_{t \mid t-1}-v_{t \mid t-2}\right)\left(\theta_{2} \varepsilon_{t-2}+v_{t \mid t-2}-v_{t \mid t-3}\right)\right] \\
\quad=\theta_{2} \omega_{1}-\theta_{2} \omega_{0}-\gamma_{0}+2 \gamma_{1}-\gamma_{2}
\end{gathered}
$$


Hence, the parameter arising from equation (1)is given by

$$
\beta=\frac{\theta_{2} \omega_{1}-\theta_{2} \omega_{0}-\gamma_{0}+2 \gamma_{1}-\gamma_{2}}{\theta_{2}^{2} \sigma^{2}+2 \theta_{2} \omega_{0}+2 \gamma_{0}-2 \gamma_{1}}
$$

This expression is the basis for an analysis of alternative special cases below, which serve illustrative purposes.

\subsection{Special cases}

\section{Econometric model only}

There are several special cases that are worth highlighting, as follows:

Case (i) $\quad F_{t \mid t-h}=M_{t \mid t-h}$

In this case, where the final forecast is just the model forecast with no intuition, such that $\gamma_{0}, \gamma_{1}, \gamma_{2}, \omega_{0}$ and $\omega_{1} \quad$ are all equal to 0 , it is clear that

$$
E\left[\left(\theta_{1} \varepsilon_{t-1}\right)\left(\theta_{2} \varepsilon_{t-2}\right)\right]=0
$$

so that $\beta=0$ in (1). This is the classic case of weak-form forecast rationality. We will now show that only in this special case of the null hypothesis $\beta=0$, does a value of $\beta$ have a straightforward and valid interpretation.

\section{Intuition only}

Case (ii) $\quad F_{t \mid t-h}=v_{t \mid t-h}$

In this case, the final forecast is based only on intuition and no model. Therefore, the forecaster does not consider the use of an econometric model, and also does not have any 
insights into the news process, $\varepsilon_{t}$, which means that $\omega_{0}$ and $\omega_{1}$ are equal to 0 . In this case, the parameter in (6) becomes

$$
\beta=\frac{-\gamma_{0}+2 \gamma_{1}-\gamma_{2}}{2 \gamma_{0}-2 \gamma_{1}}
$$

which, in turn, after dividing the numerator and denominator by $\gamma_{0}$, can be written as

$$
\beta=\frac{-1+2 \rho_{1}-\rho_{2}}{2-2 \rho_{1}}
$$

where the $\rho$ parameters are the one-period and two-period lagged autocorrelations for the intuition process. For illustrative purposes, we now consider two alternative processes for intuition, namely an autoregressive (AR) process and a moving average (MA) process:

Process (a): When intuition follows an $\operatorname{AR}(1)$ process with parameter $\lambda$, then $\rho_{1}=\lambda$ and $\rho_{2}=\lambda^{2}$. Note that intuition can have non-zero mean, which does not matter for these derivations. Substituting these two terms into equation (7) gives

$$
\beta=\frac{\lambda-1}{2}
$$

Clearly, when intuition is a stationary $\operatorname{AR}(1)$ process, that is, when $|\lambda|<1$, then $-1<\beta<0$. If intuition is simply a white noise process, then the estimate of $\beta$ equals -0.5 . For an $\mathrm{AR}(2)$ process for intuition similar results can be derived.

Process (b): When intuition follows an MA(1) process, with parameter $\theta$, then $\rho_{1}=\frac{\theta}{1+\theta^{2}}$ and $\rho_{2}=0$. Substituting these terms into equation (7) gives 


$$
\beta=\frac{-(\theta-1)^{2}}{2\left(\theta^{2}-\theta+1\right)}
$$

In Figure 1, we present the parameter, $\beta$, as a function of $\theta$. Again, it is clear that $\beta$ is negative unless $\theta=1$.

\section{Forecasts based on model and intuition}

Case (iii) $\quad F_{t \mid t-h}=M_{t \mid t-h}+v_{t \mid t-h}$, with $\omega_{0}=0$ and $\omega_{1}=0$.

In this case, where there is no correlation between current and past news and current intuition, the expression for $\beta$ is

$$
\beta=\frac{-\gamma_{0}+2 \gamma_{1}-\gamma_{2}}{\theta_{2}^{2} \sigma^{2}+2 \gamma_{0}-2 \gamma_{1}}
$$

When a time series process is postulated for intuition, it is easy to see using (7) that the value of $\beta$ is also negative. This is an interesting result as it shows that NO smoothing (Definitions 1a and 1b) and also NO over-reacting (Definitions 2a and 2b) can generate a negative value of $\beta$. This would seem to cast serious doubt on the prevailing consensus in the literature regarding the interpretation of $\beta$ when it is found empirically not to be equal to 0 .

In order to give an impression of which values of $\beta$ can emerge for the cases where (iv) $\omega_{0} \neq 0$ and $\omega_{1}=0$ and (v) $\omega_{0} \neq 0$ and $\omega_{1} \neq 0$, we consider Figure 2. There we show values of $\beta$ for the case where $\gamma_{0}=1, \gamma_{1}-0.8 \gamma_{2}=0.6$ (mimicking an AR(2) process), $\sigma^{2}=1, \omega_{0}=0,0.5$ or 0.9 , and where $\omega_{1}$ ranges from -1 to 1 . Clearly, when $\omega_{0}$ and $\omega_{1}$ are both associated with "smoothing" (whereby they are both negative), the value of $\beta$ can still be negative. In short, this is a case where there is both contemporaneous and dynamic smoothing, but the literature would typically interpret a negative value of $\beta$ as 
over-reaction. Figure 2 shows that any value of $\beta$ is possible for virtually any positive or negative values of $\omega_{0}$ and $\omega_{1}$.

In summary, when there is no correlation between current and lagged news and current intuition (Case (iii) above), then $\beta<0$. When there is a negative correlation between current news and current intuition, and when there is a positive correlation between past news and current intuition, then $\beta>0$. In the event that $\beta=0$ this can be associated with the situation (a) where the forecaster relies fully on an econometric model and also (b) where the forecaster relies fully on intuition, and where the time series properties of intuition are a random walk (that is, $\lambda=1$ in equation (8)). In contrast, when only intuition is used and intuition is a white noise process (that is, $\lambda=0$ in equation (8)), then $\beta=-0.5$.

Interestingly, and most importantly, the above derivations and definitions show that the estimated value of $\beta$ is not directly associated with smoothing or over-reaction, but rather depends heavily on the signs and values of both $\omega_{0}$ and $\omega_{1}$.

\section{Interpreting Table 1}

Using the results in the previous section, we can now evaluate the empirical results given in Table 1. It seems that theoretically the values of $\beta$ can range from around -1 to $+\infty$, with values in the range -0.5 to slightly greater than zero are most common.

A value for $\beta$ of -0.5 would mean that the forecaster may have discarded the outcome of the model, and has used expert intuition, with the peculiar property that there is zero correlation between $v_{t \mid t-h}$ and $v_{t \mid t-(h+1)}$. This absence of correlation seems quite unusual, as the intuition-based forecasts are concerned with the same fixed event. 
Dovern and Weisser (2011, p. 463) interpret a negative value of $\beta$ as a sign of overreaction, "i.e., at first, they (forecasters) revise their forecasts too much, then they undo part of this revision during the next forecasting round". Hence, they assume that $\omega_{0}>0$ and $\omega_{1}<0$. The results in the previous section show that there can also be several other situations that lead to negative values of $\beta$, specifically the covariance of current and lagged news to the numerical forecasts with current intuition.

A large and positive value of $\beta$ must mean that forecasters take current and one-period lagged news into account when forming their intuition. A negative correlation between current news and current intuition $\left(\omega_{0}<0\right)$ means that a forecaster downplays the relevance of current news, that is, there is under-reaction. This could be associated with a forecaster's uncertainty with the most recent releases of data. A positive correlation between one-period lagged news and current intuition $\left(\omega_{1}>0\right)$ suggests that the forecaster amplifies a recent shock, which might not be there, and hence over-adjusts the model forecast. In the literature, these situations are all presented under the label of "forecast smoothing".

The results in the previous section suggest that, based only on estimates of $\beta$, these separate cases cannot be disentangled, which leads to the key issue of identification. Various parameter configurations of $\gamma_{0}, \gamma_{1}, \gamma_{2}, \omega_{0}, \omega_{1}$, and especially of $\omega_{0}$ and $\omega_{1}$, can lead to various values of positive and negative $\beta$.

By far, the optimal value of $\beta$ is 0 . This could mean either that the forecaster has relied fully on an econometric model, or that the forecast is given as

$$
F_{t \mid t-h}=v_{t \mid t-h}
$$

with 


$$
v_{t \mid t-h}=v_{t \mid t-(h+1)}+\varsigma_{t}
$$

where $\varsigma_{t} \sim\left(0, \eta^{2}\right)$ is a white noise process.

What is certain, though, is that, when there is no correlation between news and intuition, it follows that $\beta$ is negative. For $\beta$ to be positive, a forecaster should under-react to current news and over-react to past news. The latter case seems to occur most frequently in practice (see Table 1).

In order to derive what forecasters actually do from the data on numerical values of $F_{t \mid t-h}$, it is necessary to obtain estimates of the news process and of intuition. This requires fitting an econometric time series model for $y_{t}$, the numerical forecast of interest, to obtain estimates of $\varepsilon_{t}$. Next, this model can be used to create estimates of the modelbased forecasts, $M_{t \mid t-h}$ and, with these, one can estimate a time series model with observations on intuition, $v_{t \mid t-h}$. These two estimated series could then be used to compute the correlations between current intuition and both current and past news. As such, one can obtain estimates of the key parameters, $\gamma_{0}, \gamma_{1}, \gamma_{2}, \omega_{0}, \omega_{1}$, and then sensibly interpret the value of the estimated $\beta$. As the variables are generated regressors, Franses, McAleer and Legerstee (2009) recommend using Newey-West HAC standard errors to correct for the measurement errors in the estimated variables..

\section{Conclusion}

This paper has shown that the interpretation of $\beta$ in a regression of forecast revisions on previous forecast revisions is not entirely straightforward. Currently, the literature unequivocally assigns meanings such as smoothing, and over-reaction or under-reaction, to positive and negative values of $\beta$, but we have shown in this paper that these are not one-to-one relationships. 
The approach developed in the paper is based on the assumption that numerical forecasts could be decomposed into both an econometric model and random expert intuition. We proposed a methodology to be able to interpret such non-zero correlations in a straightforward and clear manner. In particular, we showed that the interpretation of the sign of the correlation between the current and one-period lagged forecast revisions depends on the process governing intuition, and the current and lagged correlations between intuition and news (or shocks to the numerical forecasts). It follows that the estimated non-zero correlation cannot be given a direct interpretation in terms of smoothing or over-reaction. It was also shown that smoothing and over-reaction, modelled and interpreted correctly, can change over time.

When estimates of $\gamma_{0}, \gamma_{1}, \gamma_{2}, \omega_{0}, \omega_{1}$ are available, it also seems possible to examine the validity of other reasons for forecast updates not to be weak-form efficient, or rational. Recent work in Ashiya (2003), Amir and Ganzach (1998), and DellaVigna (2009) sketch various reasons for non-rationality. It would be interesting to examine whether professional forecasters have certain forecasting styles. We postpone such an extensive analysis for future research. Then it would be relevant to compare the behavior with the actual performance of the forecasters. Indeed, as Franses and Legerstee (2010) have shown, in order to evaluate forecast accuracy properly, one needs to know how the forecasts were actually created.

It is possible that serial correlation in intuition is an indication of lack of rationality. On the other hand, forecasters might notice that model forecasts lack information (due to a very recent shock or institutional change), and then it may be perfectly rational to include a series of adjustments. Further work on this issue is sure to shed important light on forecast rationality. 


\section{Table 1: Estimation Results for Variants of Equation (1)}

\section{Source}

Clements (1997)

Table 1, p. 233
$-0.414$

$-0.232$

0.396

0.212

Isengildina et al. (2006)
Table 2, p. 1097

Dovern and Weisser (2011)

Table 4, p. 463

$-0.040$

0.001

$-0.021$

Ager et al. (2009)

Tables 5 and 6 , pp. $178-179$

Isiklar et al. (2006)

Table II, p. 710

0.163

0.330
Estimates of $\beta$, with averaging or pooling

(average across 5 cases, GDP)

(average across 5 cases, inflation)

(average across 5 cases, Corn)

(average across 5 cases, Soybeans)

(average across G7, GDP)

(average across G7, inflation)

(average across G7, industrial production)

(average across G7, private consumption)

(average across 12 countries, GDP)

(average across 12 countries, inflation)

(pooled estimated across 18 countries, GDP)

Ashiya (2006)

Loungani (2001)

Berger and Krane (1985) often $>0$

(IMF, OECD forecasts, GDP and inflation)

often $>0$

(Consensus forecasts, 63 countries, GDP)

often $>0 \quad$ (DRI, Chase forecast, US, GNP) 


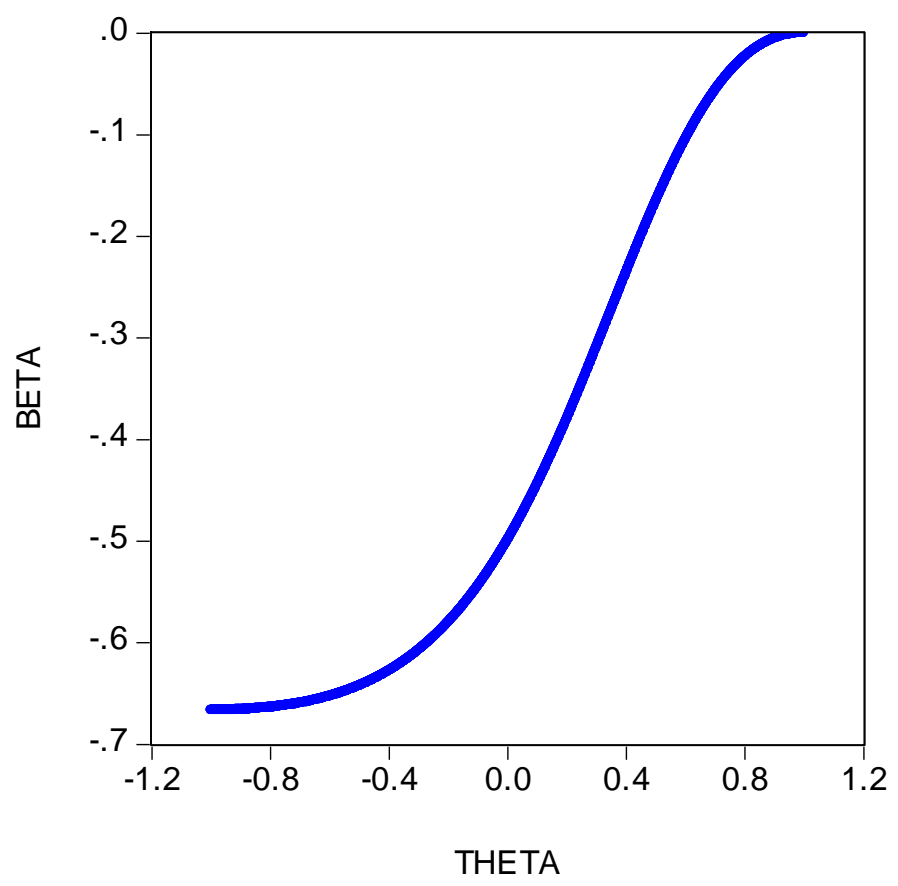

Figure 1: Relation between the parameters $\theta$ and $\beta$ for an MA(1) process underlying intuition 


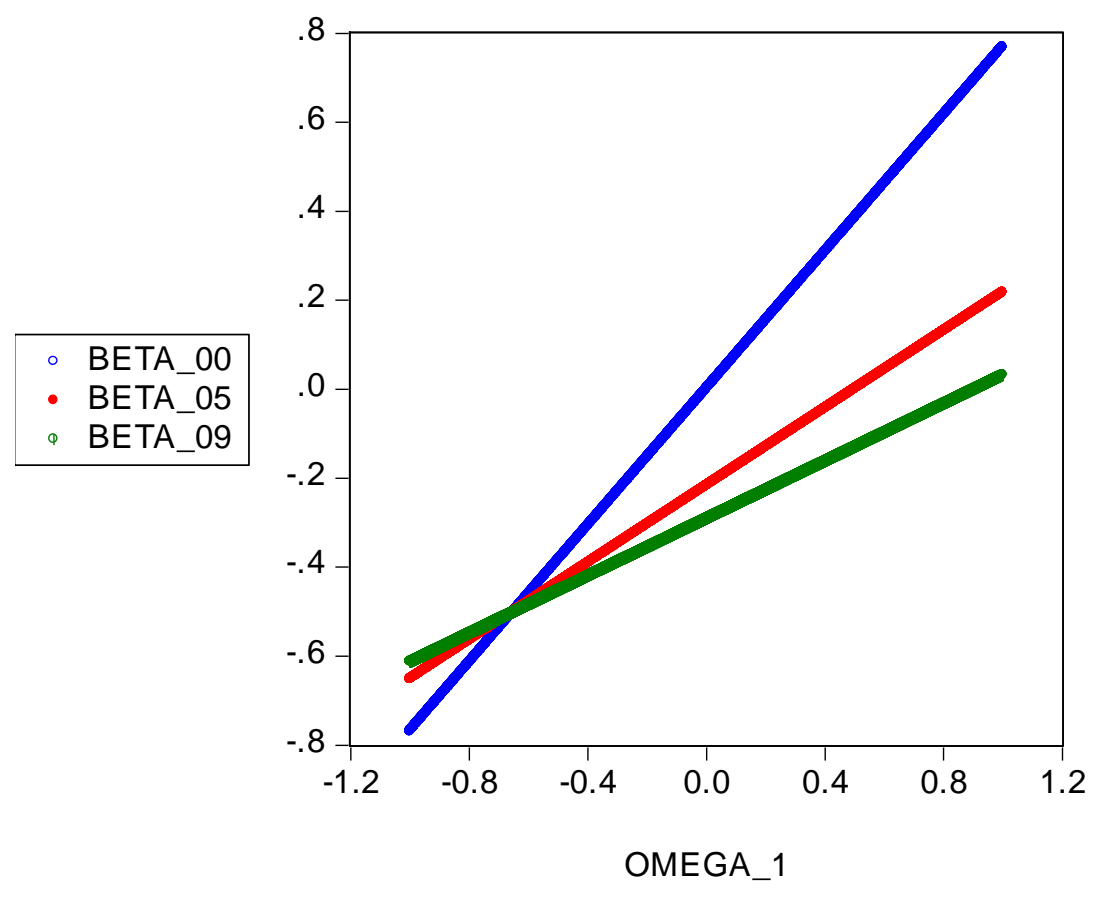

Figure 2: Values of $\beta$ when $\gamma_{0}=1, \gamma_{1}-0.8 \gamma_{2}=0.6$ (mimicking an AR(2) process), $\sigma^{2}$ $=1, \omega_{0}=0$ (BETA_00), $0.5\left(\right.$ BETA_05) or 0.9 (BETA_09), and $\omega_{1}$ ranges from -1 to 1 .

\section{References}


Ager, P., M. Kappler, and S. Osterloh (2009), The accuracy and efficiency of the Consensus forecasts: A further application and extension of the pooled approach, International Journal of Forecasting, 25, 167-181.

Amir, E. and Y. Ganzach (1998), Overreaction and underreaction in analysts' forecasts, Journal of Economic Behavior \& Organization, 37, 333-347.

Ashiya, M. (2003), Testing the rationality of Japanese GDP forecasts: The sign of forecast revision matters, Journal of Economic Behavior \& Organization, 50, 263-269.

Ashiya, M. (2006), Testing the rationality of forecast revisions made by the IMF and the OECD, Journal of Forecasting, 25, 25-36.

Berger, A.N. and S.D. Krane (1985), The informational efficiency of econometric model forecasts, Review of Economics and Statistics, 67, 128-134.

Capistran, C. and A. Timmermann (2009), Disagreement and biases in inflation expectations, Journal of Money, Credit and Banking, 41, 365-396.

Chang, C.-L., P.H. Franses and M. McAleer (2011), How accurate are government forecasts of economic fundamentals? The case of Taiwan, International Journal of Forecasting, 27(4), 1066-1075.

Cho, D.W. (2002), Do revisions improve forecasts?, International Journal of Forecasting, $18,107-115$.

Clements, M.P. (1997), Evaluating the rationality of fixed-event forecasts, Journal of Forecasting, 16, 225-239. 
DellaVigna, S. (2009), Psychology and economics: Evidence from the field, Journal of Economic Literature, 47, 315-272.

Dovern, J. and J. Weisser (2011), Accuracy, unbiasedness and efficiency of professional macroeconomic forecasts: An empirical comparison for the G7, International Journal of Forecasting, 27, 452-465.

Franses, P.H., H.C. Kranendonk and D. Lanser (2011), One model and various experts: Evaluating Dutch macroeconomic forecasts, International Journal of Forecasting, 27, 482-495.

Franses, P.H. and R. Legerstee (2010), Do experts adjustments on model-based SKUlevel forecasts improve forecast quality?, Journal of Forecasting, 29, 331-340.

Franses, P.H., M. McAleer and R. Legerstee (2009), Expert opinion versus expertise in forecasting, Statistica Neerlandica, 63, 334-346.

Isengildina, O., S.H. Irwin, and D.L. Good (2006), Are revisions to USDA crop production forecasts smoothed?, American Journal of Agricultural Economics, 88, 10911104.

Isiklar, G., K. Lahiri and P. Loungani (2006), How quickly do forecasters incorporate news? Evidence from cross-country surveys, Journal of Applied Econometrics, 21, 703725.

Laster, D., P. Bennett and I.S. Geoum (1999), Rational bias in macroeconomic forecasts, Quarterly Journal of Economics, 114, 293-318.

Lawrence, M. and M. O'Connor (2000), Sales forecasting updates: How good are they in practice?, International Journal of Forecasting, 16, 369-382. 
Loungani, P. (2001), How accurate are private sector forecasts? Cross-country evidence from consensus forecasts of output growth, International Journal of Forecasting, 17, 419432.

Nordhaus, W.D. (1987), Forecasting efficiency: Concepts and applications, Review of Economics and Statistics, 69, 667-674. 